\title{
Diabetes Mellitus Tipo 1 y Psiquiatría Infanto-Juvenil
}

\author{
FELIPE ZÚÑIGA H. ${ }^{1}$, CARLA INZUNZA C. ${ }^{2}$, CONSTANZA OVALLE G. ${ }^{3}$, TAMARA VENTURA W. ${ }^{4}$ \\ 1. Residente de Psiquiatría. Departamento de Psiquiatría, Pontificia Universidad Católica de Chile. \\ 2. Psiquiatra Infanto-Juvenil. Departamento de Psiquiatría, Pontificia Universidad Católica de Chile. \\ 3. Residente de Fisiatría. Departamento de Medicina Física y Rehabilitación. Hospital José Joaquin Aguirre, \\ Facultad de Medicina, Universidad de Chile. \\ 4. Interna de Medicina. Facultad de Medicina, Pontificia Universidad Católica de Chile.
}

\section{ABSTRACT}

\section{Type 1 Diabetes Mellitus and Child-Adolescent Psychiatry}

Objective: To review the psichological effects of DM1 on children and adolescents, and review a clinical case. Method: SIBUC (Electronic System of PUC Lybrary) Bibliographic search. Clinical Case: 13 year old adolescent diagnosed with DM1 since the age of 3, with poor treatment adherence, and poor social relationships. History of Anxiety Disorders diagnosed at age 10, partially treated with medications and psychotherapy. Currently anxious, hopeless regarding illness outcome. Treated with Sertraline and psychotherapy, presented behavioral and autonomy issues. Review: The relationship between DM1 and psychiatric topics is reviewed, including some hypotheses, issues such as attachment, body-self relationship, separation-individuation. Suggestions are made for parents and treatment teams for systematic followup of these patients and in preventing and treating psychological complications of DM1. Conclusions: DM1 is associated to significant psychopathology in children and adolescents. Due to its complications, it needs to be prevented and treated promptly to improve the outcome of these patients.

(Key words: Type 1 diabetes mellitus, pscopathology).

Rev Chil Pediatr 2009; 80 (5): 467-474

\section{RESUMEN}

Objetivo: Revisar los efectos psicológicos de la DM1 sobre los niños y adolescentes; comentar un caso clínico. Métodos: Búsqueda en la base de datos de SIBUC (Sistema Electrónico de Bibliotecas de la Pontificia Universidad Católica de Chile) de artículos que incluyeran "Diabetes Mellitus Insulino Dependiente" y "Patología Psiquiátrica en la Infancia y Adolescencia". Caso clínico: Adolescente de 13 años con diagnóstico de DM1 desde los 3 años, con mala adherencia al tratamiento y problemas relacionales. Antecedentes de trastorno ansioso, diagnosticado a los 10 años, cuya terapia, farmacológica y terapéutica, fue parcialmente cumplida. Al consultar, angustiado y desesperanzado respecto al pronóstico de su

Trabajo recibido el 18 de julio de 2008, devuelto para corregir el 17 de noviembre de 2008, segunda versión el 01 de diciembre de 2008, tercera versión el 10 de diciembre de 2008, aceptado para publicación el 18 de mayo de 2009.

Correspondencia a:

Dr. Felipe Zúñiga $\mathrm{H}$.

E-mail: felipezh@gmail.com 
enfermedad. Se inició Sertralina y psicoterapia, con regular evolución, presentando problemas conductuales y de autonomía. Revisión: Se revisa la asociación entre DM1 y patología psiquíatrica, la hipótesis de la génesis de estas asociaciones y temas como el apego, la relación cuerpo-self y la individuación. Se realizan sugerencias para los padres y para el equipo médico, respecto del seguimiento sistemático de estos pacientes, y así contribuir a una mejor prevención y tratamiento de la psicopatología asociada a la DM1. Conclusión: La DM1 se asocia a importante psicopatología en la infancia y adolescencia. Por sus importantes implicancias esta debe ser estudiada en mayor profundidad y la enfermedad prevenida y tratada oportunamente para mejorar el abordaje de estos pacientes.

(Palabras clave: Diabetes mellitus tipo 1, psicopatología).

Rev Chil Pediatr 2009; 80 (5): 467-474

\section{Introducción}

La Diabetes Mellitus 1 es una patología crónica caracterizada por glicemias elevadas en forma persistente producida por una disfunción de células pancreáticas productoras de insulina. Los pacientes aquejados por esta enfermedad están sometidos a una serie de factores estresantes, dados por modificaciones drásticas de estilos de vida, hábitos de autocuidado y el enfrentamiento de complejas técnicas de tratamiento y control, todo lo cual deviene en un evidente riesgo de inestabilidad en su salud mental.

Recibir el diagnóstico de DM1 en la infancia $o$ en la adolescencia pone en riesgo el normal desarrollo psicológico del paciente y del ciclo evolutivo familiar, cuyas consecuencias podrán hacerse evidentes tanto a nivel de su personalidad, como en la funcionalidad de la familia ${ }^{1}$. Pese a esta evidencia, las implicancias psicológicas de la DM1 en niños y adolescentes han sido escasamente estudiadas desde una perspectiva integral. Sin embargo, se ha asociado la aparición de diversos trastornos psiquiátricos en estos pacientes. Los más observados son los adaptativos y afectivos y, en menor medida, trastornos ansiosos, alimentarios, de pánico, de personalidad (limítrofe, antisocial), somatomorfos y trastornos psicóticos. La capacidad adaptativa al stress, el apego seguro y un desarrollo sano de la personalidad y de las relaciones interpersonales disminuyen la incidencia de morbilidad psiquiátrica en estos pacientes ${ }^{2-5}$.

El presente artículo intenta reflexionar en base a la revisión de literatura ad-hoc y análisis de un caso clínico, acerca de la asociación entre DM1 y la psicopatología y evaluar además en que sentido interfiere la DM 1 en el desarrollo emocional de los pacientes. Para ello se profundiza en la superación de las angustias básicas, en especial la de separación, debido a los conflictos en el apego a los que predispone esta patología crónica.

\section{Método}

Se realizó durante los meses de julio y agosto de 2007 una revisión no sistemática de la literatura disponible en el Sistema Electrónico de Bibliotecas de la Pontificia Universidad Católica de Chile (SIBUC), incluyendo en la búsqueda los conceptos: Diabetes Mellitus tipo 1, Insulino Requirente o Dependiente y Patología Psiquiátrica en Infancia o Adolescencia. Se incluyó en la revisión final un total de 15 artículos que dieron cuenta de la relación entre esta citada patología y condiciones psicopatológicas. Adicionalmente se realizó análisis en profundidad de ficha clínica y 2 entrevistas no estructuradas (individuales y familiares) a un paciente con diagnóstico de DM1 atendido en la Red de Salud de la Pontificia Universidad Católica de Chile. Dichas entrevistas cumplieron el protocolo utilizado en el servicio de psiquiatría que evalúa, además de la historia clínica, la apariencia, conducta, actitud, contacto, conciencia, pensamiento, afecto, memoria, capacidad intelectual, insight e ideación suicida. No se aplicaron otros test psicológicos.

\section{Resultados}

El término Diabetes proviene del griego 
diabaino ("caminar"), formado a partir del prefijo dia- ("a través de") y baíno ("andar, pasar"). Areteo de Capadocia (Siglo I DC) fue el primero en utilizarlo etimológicamente como "tránsito, paso", aludiendo a la excesiva expulsión de orina, la poliuria, característica de esta enfermedad, dando origen al sentido de "irse por la orina". Siguiendo a esta descripción histórica, conocemos en la actualidad al menos dos clases de diabetes, la mellitus (caracterizada por "orinas dulces") y la insípida ${ }^{6,7}$.

La Diabetes Mellitus es una patología crónica caracterizada por glicemias elevadas en forma persistente, producida por disfunción de células pancreáticas productoras de insulina y/o por una dificultad celular periférica en incorporar la glucosa. La DM1, conocida también como Insulino Dependiente, corresponde al primer grupo citado y se define por un déficit absoluto de insulina y, en consecuencia, por una dependencia vital a su administración exógena ${ }^{1,8}$.

La Diabetes Mellitus que aparece en menores de 20 años corresponde en un 84\% a DM1. Su presentación puede ocurrir a cualquier edad, pero se da con mayor frecuencia en menores de 15 años, con 2 peaks de presentación entre los 4 a 6 años y los 12 y 14 años. Existe un tercer peak de presentación entre los 50 y 60 años, clasificados como diabetes tipo LADA (Late Autoinmune Diabetes of the Adult). La etiología es en el 90\% de los casos autoinmune. El 10\% restante se clasifica como idiopático sin asociación de marcadores de autoinmunidad ${ }^{8}$.

La incidencia de DM1 en el mundo es variable: China y Venezuela presentan 0,1 casos nuevos (cada año por cada 100000 habitantes menores de 15 años), mientras que en Finlandia e Italia cuentan con hasta $36,5 \operatorname{casos}^{8}$. Chile ha presentado tasa de incidencia en progresivo incremento, contando, para el año 2004 con un 8,33/100 000 casos nuevos por cada 100000 habitantes menores de 15 años ${ }^{9}$.

En relación a su expresión clínica, la hiperglicemia sostenida produce complicaciones agudas (cetoacidosis) y de largo plazo (especialmente secundarias a microangiopatías) como retinopatía, neuropatías y nefropatía, además de complicaciones cardiovasculares ${ }^{8,9}$. Respecto a las primeras, existe mayor riesgo de su apari- ción (y con pródromo más breve) en función de la precocidad del debut diagnóstico ${ }^{2-4,8-11}$. El manejo de estos pacientes de menor edad es, asimismo, particularmente difícil por la gran variabilidad en su nivel de actividad física, ingesta alimentaria e infecciones concomitantes $^{2-4,8-11}$.

Los pacientes aquejados por esta enfermedad están sometidos a una serie de factores estresantes, dados por modificaciones drásticas de estilos de vida (dieta, ejercicio), hábitos de autocuidado y el enfrentamiento de complejas técnicas de tratamiento y control, todo lo cual deviene en un evidente riesgo de inestabilidad en su salud mental ${ }^{4-5,10-13}$. Los trastornos psiquiátricos más observados en estos pacientes (niños y adolescentes) son trastornos adaptativos y afectivos y, en menor medida, trastornos ansiosos, alimentarios, de pánico, de personalidad (limítrofe, antisocial), somatomorfos y trastornos psicóticos ${ }^{2-5,12,14}$.

El trastorno adaptativo es el diagnóstico psiquiátrico más común ${ }^{2}$. Su mayor incidencia se presenta durante el primer año de diagnóstico y se encuentra asociado significativamente a depresión ${ }^{2,15-18}$. Distintos trabajos han hallado una significativa relación de esta patología con mal control metabólico ${ }^{2-4,18}$. En relación a depresión, su prevalencia es 2 a 3 veces mayor que en la población general, con peaks de incidencia durante el primer y décimo año desde el diagnóstico (eventualmente secundario a limitación laboral concurrente al comienzo de la vida adulta) ${ }^{14-18}$. La relación entre DM2 (no insulino-dependiente) y depresión se encuentra mejor estudiada que con DM1.

Las hipótesis planteadas dicen relación con aumento de actividad simpática y una respuesta alterada a estresores ambientales y al incremento plasmático de catecolaminas, con la consiguiente desregulación metabólica ${ }^{1,15}$. Por otra parte, se ha estudiado la relación entre depresión y sistema neuroendocrino ${ }^{1,16}$, hallándose que el $70 \%$ de los adolescentes con DM1 produce anticuerpos anti GAD (descarboxilasa de ácido glutámico) lo cual afecta la síntesis de GABA en la célula beta pancreática y el sistema nervioso central. Estos anticuerpos jugarían un rol en el desarrollo de DM1 y la depresión. Por otro lado, las hormonas contrarreguladoras 
como el cortisol, liberado en períodos de estrés, favorecerían un peor control metabólico en estos pacientes ${ }^{17,18}$ (figura 1).

Un aspecto de relevancia es el riesgo suicida en pacientes DM1 con diagnóstico de depresión (10 veces mayor riesgo de ideación e intentos que el resto de la población, incluyendo depresivos no diabéticos), en especial considerando el acceso que estos tienen a un eficaz método de autolisis como es la administración de insulina ${ }^{14-16}$.

Los trastornos ansiosos corresponden al tercer desorden psiquiátrico más frecuente, generalmente asociados -como comorbilidad- a depresión ${ }^{1,14}$. Distintas investigaciones no han encontrado una relación significativa de esta psicopatología con el control metabólico ${ }^{1,14}$.

En cuanto a los trastornos alimentarios, en estos pacientes se presenta una mayor prevalencia de anorexia nerviosa y bulimia ${ }^{2-4}$, consi- derándose además el efecto anabólico de la insulina, que lleva a muchos de estos pacientes a suspender el tratamiento para evitar el aumento de peso $^{2-5}$.

Por otra parte, el importante impacto de esta patología en el sistema familiar del paciente, planteándose ciertos patrones comunes de disfuncionalidad como detrimento en habilidades de enfrentamiento del estrés y problemas en el cuidado temprano ${ }^{13}$, temas a analizar en profundidad en la discusión de este mismo artículo.

En relación al tratamiento, se han desarrollado pocos estudios sobre manejo específico de patología psiquiátrica en DM1. La capacidad de adaptación familiar es crítica, por lo que terapias conductuales-familiares, serían efectivas para prevenir y tratar la psicopatología en estos jóvenes ${ }^{10,11,13,19,20}$. Se recomienda consulta psiquiátrica precoz y apoyo en pares o con

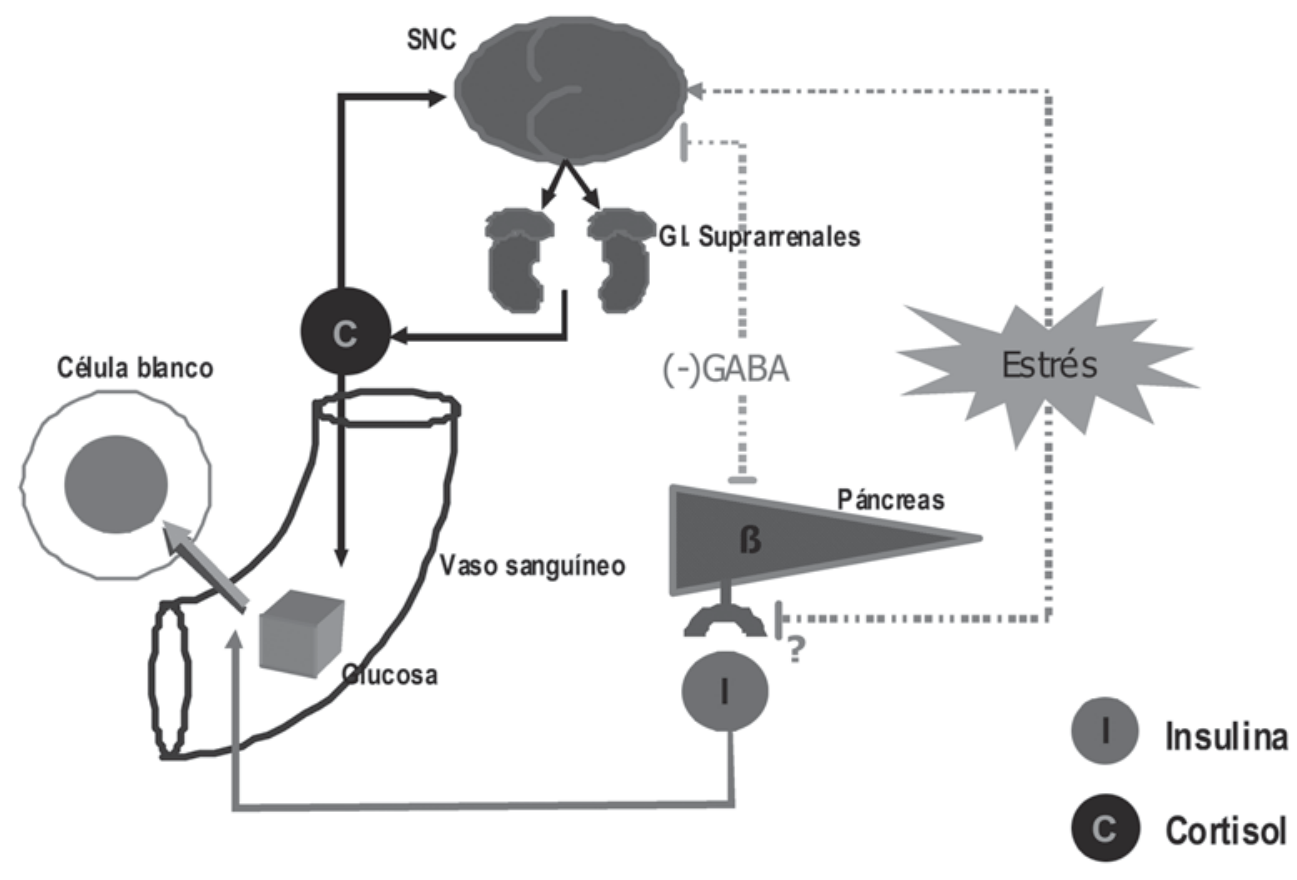

Figura 1. Depresión y Diabetes Mellitus 1. Mecanismos propuestos. Efectos del estrés sobre el metabolismo de la glucosa (mediante estimulación de secreción suprarrenal de cortisol y eventual inhibición de secreción pancreática de insulina) y sobre perfomance anímica (mediante impacto cerebral vía hiper-cortisolemia). 
grupos de ayuda, los cuales en el caso de diabetes juvenil se encuentran muy bien organizados tanto en Chile como en otros países ${ }^{21}$.

Sobre el tratamiento farmacológico, la evidencia relevante señala ciertas precauciones con los antidepresivos inhibidores de la monoamino-oxidasa (hipoglicemia) y antidepresivos tricíclicos (hiperglicemia). Los antidepresivos inhibidores de la recaptación de serotonina tienden a aumentar la sensibilidad periférica a insulina, en especial la fluoxetina y la sertralina. Esta última cuenta con la ventaja adicional de una menor interacción metabólica hepática, por lo cual es una excelente opción para manejo de los cuadros depresivos y ansiosos en estos pacientes $^{16-18}$.

\section{Caso Clínico}

Se trata de un paciente de 13 años de sexo masculino, con diagnóstico de DM1 desde los 3 años de edad, que fue derivado desde la Unidad de Nutrición y Diabetes de la Red de Salud UC a Unidad de Psiquiatría Infantil de la misma red. El motivo de consulta dice relación con mal control metabólico, asociado a importantes dificultades en adherencia terapéutica y emergencia de complicaciones expresadas en una incipiente falla renal.

El paciente es el segundo hijo de un matrimonio de edad media. La primera hija murió a los 6 años de edad producto de una patología neurodegenerativa (Leucodistrofia Metacromática), cuando el paciente tenía 1 año de edad. La madre señala que este proceso implicó evidentes faltas de cuidado al hijo en cuestión en virtud de la sobredemanda de la hija enferma y posterior duelo materno (padres señalan que este período fue "devastador", "seguimos adelante sólo por él"). Transcurridos tres años de este deceso, nació la tercera hija del matrimonio.

La madre señala que el embarazo del paciente fue deseado, en contexto de consejo genético por patología de hija. "Fue un embarazo delicado" por citadas demandas de cuidados extremos de hija mayor. Sin antecedentes neonatales de relevancia. Descrito como un niño "inquieto, pero no tan mañosito". Lac- tancia materna hasta los 3 meses, con incremento ponderal adecuado. Hitos del desarrollo sin alteraciones significativas, comienza a asistir a jardín infantil a los 3 años, mismo tiempo en que se le realiza diagnóstico de DM1 como eventual complicación de cuadro respiratorio grave. Al iniciar la vida escolar (pre-kinder), la madre refiere importantes dificultades de adaptación que comienzan a ser más intensas a partir de tercero básico: "empezó a ponerse inquieto, desordenado, distraído, a veces muy nervioso y conflictivo". Con rendimiento escolar regular, no presentó repitencias ni cambios de colegio. No se refieren consultas a salud mental.

A los 10 años de edad se decide finalmente consultar a psiquiatra quien diagnosticó trastorno ansioso iniciándose manejo con citalopram. Asimismo, se sugirió intervención familiar y psicoterapia individual. La familia y el paciente presentan cumplimiento discreto de terapéutica indicada.

A los 13 años es nuevamente derivado a salud mental por el médico tratante de DM1 para lograr adherencia a tratamiento y mejoría de parámetros metabólicos en contexto de una incipiente repercusión multisistémica del cuadro. Al momento de la consulta y en relación a su enfermedad, el paciente presentaba altos niveles de angustia, refiriendo: "lo más fome es pincharme todos los días". Asimismo, señala haber cursado con sentimientos de desesperanza y "desgano" frente al pronóstico de su enfermedad y ante importante limitación vital (especialmente detrimento social). Pese a mostrar conciencia de las consecuencias de tratamiento irregular, no se aprecia mayor motivación para el cambio conductual requerido. "Lo único que deseo es no ser diabético" y no ir al colegio "para que mis amigos no me vean pincharme".

Durante las primeras evaluaciones el paciente impresionaba esencialmente tranquilo, medianamente cooperador (ocasionalmente con actitudes de oposición pasiva ante el entrevistador), aunque con ánimo depresivo y por momentos irritable. Se apreciaban, asimismo, rasgos de timidez y dificultad en la verbalización de conflictos. Respecto a conciencia de enfermedad, el paciente esboza preocupación por 
detrimento anímico y ante dificultad del seguimiento de indicaciones médicas. Sin otros hallazgos significativos en el examen mental. No se realizaron otras pruebas psicodiagnósticas al paciente en cuestión.

Se le diagnostica un trastorno ansioso-depresivo, iniciándose manejo con Sertralina a dosis de $25 \mathrm{mg} /$ día. El paciente evoluciona regularmente, con persistencia de dificultades en el cumplimiento de manejo de su patología de base. Asimismo, se detecta importante conflictividad familiar, emergiendo en este contexto elementos claramente disfuncionales que motivan intervención terapéutica específica para lograr mayor contención desde el entorno. En la familia se observa una importante falta de complementaridad en los roles parentales y dificultad para tolerar la necesaria mayor autonomía que el paciente reclama respecto de su etapa de desarrollo. Por otra parte, el paciente busca hacer valer su autonomía pero evita hacerse cargo de la enfermedad, manifestando una evidente negación y evitación del conflicto. Se constata, asimismo, la presencia de conductas límites (hurto, mentiras y agresividad sostenida hacia sus progenitores) que posteriormente paciente vivencia con culpa y arrepentimiento.

\section{Discusión}

La relación entre la Diabetes Mellitus 1 y la patología psiquiátrica ha sido escasamente estudiada. Sin embargo, se han asociado los trastornos adaptativos y afectivos y, en menor medida, trastornos ansiosos, alimentarios, de pánico, de personalidad (limítrofe, antisocial), somatomorfos y trastornos psicóticos a esta enfermedad crónica ${ }^{2-5,12,14}$.

La Diabetes Mellitus 1 tiene dos peaks de presentación, entre los 4 a 6 años y los 12 y 14 años, que coinciden con el desarrollo emocional/superación de angustias básicas y generación de la autonomía e individuación del niño respectivamente ${ }^{22,23}$. En el caso analizado, la Diabetes se diagnostica en el primer peak, y las descompensaciones metabólicas se producen predominantemente en la adolescencia. Analizaremos las posibles hipótesis de la génesis de este trastorno y de las descompensaciones a la luz de la revisión realizada y el caso clínico y posteriormente sugeriremos algunas acciones preventivas y terapéuticas al respecto.

Surgen de la revisión bibliográfica varias hipótesis respecto a las causas de descompensación metabólica de estos pacientes, tanto biológicas como psicológicas. Desde el punto de vista biológico las descompensaciones metabólicas podrían ser el resultado de un aumento de la actividad simpática asociada a una respuesta alterada a los estresores ambientales. Por otro lado, conocida es la relación entre los fenómenos autoinmunes y psiquiátricos ${ }^{1,16}$ lo que podría constituirse en un círculo vicioso "productor" de dichas descompensaciones: la diabetes trae la angustia y depresión y viceversa, y esto a su vez fomenta las descompensaciones metabólicas.

Estas hipótesis debiesen ser consideradas al momento de elección del tratamiento farmacológico, evitando los inhibidores de la monoamino-oxidasa (hipoglicemia) y antidepresivos tricíclicos (hiperglicemia), prefiriendo los antidepresivos inhibidores de la recaptación de serotonina por su tendencia a aumentar la sensibilidad periférica a insulina contraponiendose a la acción de las hormonas contrarreguladoras.

Desde el punto de vista psicológico la Diabetes Mellitus puede considerarse un trastorno de la nutrición y desde esa perspectiva una alteración en el apego. "Comer" es una conducta que está ligada al cuidado materno desde el nacimiento. El primer amor parece tener sus bases en la lactancia y los primeros cuida$\operatorname{dos}^{22,23}$. En el desarrollo normal se va estableciendo una relación alimento-cuidador que integra los niveles fisiológicos más básicos como parte de ella. En la patología del comer, estos niveles siguen juntos y no logran autonomía. El restringir, rechazar o recibir alimentos estará entonces asociado a la proto unidad alimentocuidador. No es sólo el alimento lo que se restringe o recibe, sino es también la sensación de ser cuidado ${ }^{22,23}$. En la Diabetes Mellitus y especialmente en este caso clínico, al restringir la madre el alimento como parte del cuidado de la enfermedad, el niño crece con la paradoja de que la madre para cuidarlo debe restringir el alimento y por lo tanto, el cariño. Una parte de 
él se desarrolla sintiendo que no lo quieren, no lo cuidan, no lo alimentan; la madre también siente que no entrega lo que necesita ${ }^{24}$. Los espacios de cercanía con la madre están llenos de reglas y limitaciones, que impiden que se genere un vínculo seguro. Todo esto, desde la perspectiva de un niño, que no entiende ni vivencia permanentemente la enfermedad, y sólo se relaciona con ella durante el tratamiento: al inyectarse insulina, siente el dolor y percibe la enfermedad. Asimismo, aprende que el dolor lo cuida, otra paradoja más que es internalizada como un cuidado/apego ambivalente e insegu$\mathrm{ro}^{22-24}$.

¿Cómo explicar la descompensación del caso clínico justo en la pre adolescencia? La teoría del apego de Bowlby supone que una fisiología adulta se acoplará al recién nacido para asegurarle sobrevivencia, produciéndose una empatía sobreinvolucrada que asegura la madurez fisiológica del niño ${ }^{22,23}$. Resolver la autonomía supone desacoplarse de la fisiología adulta y adquirir certeza de unidad separada. Este es el proceso de individuación que va construyendo el aparato mental de cada uno de nosotros. La biografía familiar puede entorpecer este proceso dejando a uno de los miembros atrapado, sobreinvolucrado con otro como si la vida de ambos dependiera de la existencia de éste ${ }^{22,23}$.

En el caso analizado, el niño siente que es educado de acuerdo a las necesidades de su madre, más que a las propias. Esto debido a su enfermedad, que produce un ambiente, resguardado por la madre, que norma y limita la nutrición/apego. Cuando los mensajes iniciales del niño no reciben respuestas confirmadoras y validantes, éste no puede desarrollar un sentido sano de self (si mismo), experimentándose a si mismo simplemente como una extensión de su madre, y no como un centro de autonomía con su propio derecho. Esta construcción relacional altera el funcionamiento mental y predispone, especialmente durante la adolescencia, a encontrar la separación a través de una pseudoautonomía. Es decir, se recurriría a conductas que llevan en el mediano o largo plazo, a complicaciones mayores y aparición de comorbilidades psíquicas y somáticas. Un ejemplo de esto lo podemos visualizar en la emergencia de citadas conductas límites del paciente reseñado (hurto). Resolver el apego inicial que vuelve a reactivarse en la adolescencia supondrá distinguir entre lo propio y lo próximo. Sólo así se podrá producir la conexión con "un otro" y salir de la sensación de soledad. Superar la sobreempatía y la confusión de identidades es la tarea de la adolescencia. Por ello, tomar una decisión de administración del sí mismo contraria al modo parental, es una conducta característica de la etapa y que no necesariamente conduce a patología. La enfermedad está en sostener con tenacidad la decisión de administrar el disconfort psíquico a través de conductas que llevan a un inminente riesgo vital ${ }^{23}$.

Mencionado lo anterior, creemos que el seguimiento temprano a los niños diagnosticados de DM1 promovería una estructura de personalidad y vinculación sana mediante la aceptación y comprensión de los conflictos psíquicos inherentes a esta enfermedad, lo que llevaría además a un mejor control metabólico a lo largo del tiempo. Este seguimiento debiese ser previo al inicio de la psicopatología y debiese además brindar apoyo y psicoeducación a los padres de tal manera que comprendan en profundidad a lo que estarán expuestos sus hijos y así estar más preparados para vincularse con ellos de una forma segura ${ }^{24}$.

En conclusión, la Diabetes Mellitus es una patología que compromete al individuo en forma global ${ }^{24}$. La psicopatología asociada a la Diabetes Mellitus 1 incluye los trastornos adaptativos y afectivos y, en menor medida, trastornos ansiosos, alimentarios, de pánico, de personalidad (limítrofe, antisocial), somatomorfos y trastornos psicóticos. La revisión bibliógrafica plantea distintas hipótesis, tanto biológicas como psicológicas, respecto a la génesis de la DM1 y de sus descompensaciones. Las hipótesis biológicas debiesen ser estudiadas mayormente $\mathrm{y}$ consideradas al momento de elección del tratamiento farmacológico de los trastornos psiquiátricos. Desde el punto de vista psicológico, el caso clínico analizado evidencia las dificultades físicas, psicológicas y sociales de la DM1 en la infancia y demuestra la importancia de los conflictos intrapsiquícos como predictores de la adherencia al tratamiento y en consecuencia el pronóstico de la enfermedad. Al producirse la DM1 en etapas tempranas del desarrollo, tiene 
el riesgo de dejar "huellas" que si no son tratadas a tiempo pueden causar un daño irreparable y conflictos transgeneracionales ${ }^{24}$.

La propuesta de este trabajo es realizar un seguimiento temprano a los niños diagnosticados de DM1. Las ventajas de esto serían la promoción de una estructura de personalidad y vinculación sana mediante la aceptación y comprensión de los conflictos psíquicos inherentes a esta enfermedad, lo que llevaría además a un mejor control metabólico a lo largo del tiempo. En este sentido, el seguimiento debiese ser previo al inicio de la psicopatología. Asimismo, sería importante brindar apoyo a los padres y realizar una psicoeducación sistemática, de tal manera que comprendan en profundidad a lo que estarán expuestos sus hijos durante el desarrollo de esta enfermedad y así estar más preparados para vincularse con ellos de una forma segura ${ }^{24}$.

\section{Referencias}

1.- López SG: Depresión y Diabetes. En Jadresic A, Ojeda C, Pérez G. Editores. Psiconeuroendocrinología. Ed. Mediterráneo. Cap. 8.

2.- Nakazato $M$, et al: Psychiatric disorders in juvenile patient with insuline-dependent diabetes mellitus. Diabetes Res Clin Pract 2000; 48: 177-83.

3.- Kovacs, et al: Psychiatric disorder and metabolic control among youths with IDDM. A longitudinal study. Diabetes Care 1996; 19 (4): 318-23.

4.- Vila $G$, et al: Insulin-dependent diabetes mellitus in children and in adolescents: value of pedopsychiatric follow-up. Arch Pediatr 2000; 4 (7): 615-22.

5.- Garrison $M$, et al: The impact of Psychiatric Comorbidities on Readmissions for Diabetes in Youth. Diabetes Care 2005; 28: 2150-4.

6.- Diccionario de la Real Academia de la Lengua: www.rae.es (acceso Julio 2007).

7.- Diccionario Etimólogico de la Medicina: www. dicciomed.es (acceso Julio 2007).

8.- Varios: Harrison's Principles of Internal Medicine, 16th Edition. Ed. McGraw-Hill, 2007 (acceso edición on-line vía SIBUC Julio 2007).

9.- Carrasco PE, Angel BB, Codner E, et al: Incidencia de diabetes mellitus tipo 1 en Santiago de Chile: análi- sis por comunas de la Región Metropolitana en el período 2000-2004. Rev Méd Chile 2006; 134 (10): 1258-64.

10.- Guitard-Munnich C: The adolescent and diabetes: diabetes works me up. Diabetes Metab 2001; 27 (4 Pt 2): S31-4.

11.- Tubiana-Rufi $N$ : Interdisciplinary management of the diabetic adolescent. "Between two balances". Diabetes Metab 2001; 27 (4 Pt 2): S26-30.

12.- Al-Haidar FA: Inpatient child and adolescent psychiatric referrals in Saudi Arabia: clinical profiles and treatment. East Mediterr Health J 2006; 9 (5-6): 9961002.

13.- Minuchin $S$ : The psychosomatic family in child psychiatry. J Am Acad Child Psychiatry 1979; 18 (1): 76-90.

14.- Dantzer C, et al: Anxiety and depression in juvenile diabetes: a critical review. Clin Psychol Rev 2003; 23 : 787-800.

15.- Lawrence J, et al: Prevalence and Correlates of Depressed Mood Among Youth With Diabetes: The SEARCH for Diabetes in Youth Study. Pediatrics 2006; 117: $1348-58$.

16.- Grey $M$, et al: Depression in Type 1 Diabetes in Children: Natural History and Correlates. J Psychosomat Res 2002; 53: 907-11.

17.- Lustman P, et al: Depression-related Hyperglycemia in Type 1 Diabetes: A mediational approach. Psychosomat Med 2005; 67: 195-9.

18.- Miranda A, et al: Depressed Mood is a factor in Glycemic Control in Type 1 Diabetes. Psychosomat Med 2001; 63: 551-5.

19.- Ginsburg K, et al: Parent's perceptions of Factors that affect successful Diabetes Managment for Their Children. Pediatrics 2005; 116: 1095-104.

20.- Clay D: Empirically supported treatments in pediatric psychology: where is the diversity? J Pediatr Psychol 2002; 27 (4): 325-37.

21.- Sitio Web Fundación Diabetes Juvenil de Chile: www.diabeteschile.cl. Acceso Julio 2007.

22.- Cordella P: Proposición de un modelo para comprender la configuración anoréctica. Rev Chil Pediatr 2002; 73 (6): 566-75.

23.- Cordella P: Psicoterapia: un modelo para comprender la configuración anoréctica. U. del Desarrollo, mayo, 2003, Santiago de Chile.

24.- Ismail $K$ : Eating disorders and diabetes. Psychiatry 2008; 7 (4): 179-82. 